\title{
Thermodynamics of Yukawa systems and sound velocity in dusty plasmas
}

\author{
S A Khrapak \\ Forschungsgruppe Komplexe Plasmen, Deutsches Zentrum für Luft- und Raumfahrt, Oberpfaffenhofen, \\ Germany \\ Aix-Marseille-Université, CNRS, Laboratoire PIIM, UMR 7345, 13397 Marseille cedex 20, France \\ E-mail: Sergey.Khrapak@dlr.de
}

Received 23 June 2015, revised 2 October 2015

Accepted for publication 5 October 2015

Published 9 November 2015

\begin{abstract}
The simple practical approaches to estimate thermodynamic properties of three-dimensional Yukawa systems across coupling regimes (in fluid and solid phases) are summarized. These approaches demonstrate very good accuracy when compared with the results of direct numerical simulations. To demonstrate possible applications, the sound velocity in a strongly coupled dusty plasma is evaluated by combining the conventional fluid description of multicomponent plasma with the appropriate equation of state of Yukawa fluids. Limitations of the proposed approaches are briefly discussed.
\end{abstract}

Keywords: Yukawa systems, dusty (complex) plasmas, equation of state

\section{Introduction}

The thermodynamics of Yukawa systems (particles interacting via the pairwise Yukawa repulsive potential) is of considerable interest, in particular in the context of conventional plasmas, dusty (complex) plasmas, and colloidal suspensions [1]. For small charged point-like particles, the Yukawa potential (also known as screened Coulomb or Debye-Hückel potential) reads

$$
V(r)=\left(Q^{2} / r\right) \exp (-r / \lambda),
$$

where $Q$ is the charge, $\lambda$ is the screening length associated with the neutralizing medium (normally given by the corresponding Debye radius), and $r$ is the distance between a pair of particles. The effect of the neutralizing medium on the effective interactions between the particles can involve more than only screening. This is particularly relevant in complex plasmas, where continuous absorption of plasma electrons and ions on the particle surface (for simplicity we neglect the electron emission processes, which can play a role in certain situations, and then can make the problem even more complicated) results in inverse-power-law asymptotes of interaction at large interparticle separations [2-8]. At intermediate distances deviations from the simple form (1) can be expected when ion-particle interaction is highly non-linear [9-11] or a significant fraction of trapped ions is present [12]. Plasma production and loss processes can produce a double-Yukawa interaction potential characterized by two different screening lengths [13, 14]. Nevertheless, many experimentally observed trends can be already reproduced by the simplest model, considering point-like particles interact via the repulsive Yukawa potential (1), at least qualitatively. For this reason this model, which we will refer to as the single component Yukawa system (SCYS), has received a great deal of interest.

Single component Yukawa systems are fully characterized by two dimensionless parameters. The first is the coupling parameter, $\Gamma=Q^{2} / a T$, where $a$ is the characteristic interparticle separation (in this paper we take $a=(3 / 4 \pi n)^{1 / 3}$, where $n$ is the particle density) and $T$ is the temperature (in energy units). The second is the screening parameter, defined as $\kappa=a / \lambda$. As $\Gamma$ increases at some fixed $\kappa$, the Yukawa system shows a transition from a weakly coupled gas-like regime $\Gamma \lesssim 1)$ to a strongly coupled fluid regime $(\Gamma \gg 1)$ and finally crystallizes into either bcc or fcc crystalline lattice at some $\Gamma_{\mathrm{m}}$, which depends on $\kappa$ (the subscript ' $\mathrm{m}$ ' indicates melting).

Phase diagram and thermodynamic properties of Yukawa systems have been relatively well investigated using various computational and analytical techniques. Some relevant examples include the Monte Carlo (MC) and molecular dynamics (MD) numerical simulations [15-18], as well as 
integral equation theoretical studies [19-21]. Semi-empirical fitting formulas [22, 23] and simplistic approaches [24, 25] to estimate thermodynamic properties of Yukawa systems (including the limiting case of one-component-plasma [26, 27]) have been discussed recently in the literature. Their accuracy is, however, insufficient in many cases.

The purpose of the present paper is to summarize recently proposed, convenient and practical approaches to evaluate thermodynamic properties of three-dimensional Yukawa fluids and solids in a wide region of the respective phase diagram. The approaches are based on simple phenomenological arguments, which are likely applicable to a wide class of soft repulsive interactions. They demonstrate impressive accuracy when compared with the 'exact' results from numerical simulations. As an example, relevant to Yukawa fluids, the sound velocity in a strongly coupled dusty plasma is evaluated using the conventional fluid description of multi-component plasma, supplemented by an appropriate equation of state.

\section{Model}

We consider the system of $N$ point-like particles in three dimensions, which occupy the volume $V(N, V \rightarrow \infty$ in the thermodynamic limit), have the temperature $T$ (temperature will be expressed in energy units), and interact with each other via the repulsive Yukawa potential (1). The main thermodynamic quantities of interest are the internal energy $U$, Helmholtz free energy $F$, and pressure $P$ of the system. These thermodynamic functions are related via [28]

$$
\begin{gathered}
U=-T^{2}\left(\frac{\partial}{\partial T} \frac{F}{T}\right)_{V}, \\
P=-\left(\frac{\partial F}{\partial V}\right)_{T} .
\end{gathered}
$$

In the following, we will use conventional reduced units: $u=U / N T, f=F / N T$, and $Z \equiv p=P V / N T$, where $Z$ is the compressibility factor (reduced pressure).

It is useful to express the reduced thermodynamic functions in terms of Yukawa system phase state variables, $\kappa$ and $\Gamma$. For a fixed number of particles we have $\Gamma \propto(a T)^{-1} \propto V^{-1 / 3} T^{-1}$ and $\kappa \propto a \propto V^{1 / 3}$. This implies

$$
\frac{\partial \Gamma}{\partial T}=-\frac{\Gamma}{T}, \quad \frac{\partial \Gamma}{\partial V}=-\frac{1}{3} \frac{\Gamma}{V}, \quad \frac{\partial \kappa}{\partial T}=0, \quad \frac{\partial \kappa}{\partial V}=\frac{1}{3} \frac{\kappa}{V} .
$$

The following equations for the reduced thermodynamic functions are then easily obtained:

$$
\frac{u}{\Gamma}=\frac{\partial f}{\partial \Gamma}
$$

and

$$
Z=1+\frac{\Gamma}{3} \frac{\partial f}{\partial \Gamma}-\frac{\kappa}{3} \frac{\partial f}{\partial \kappa}
$$

we consider (except when specially mentioned), the single component Yukawa system, where thermodynamics is

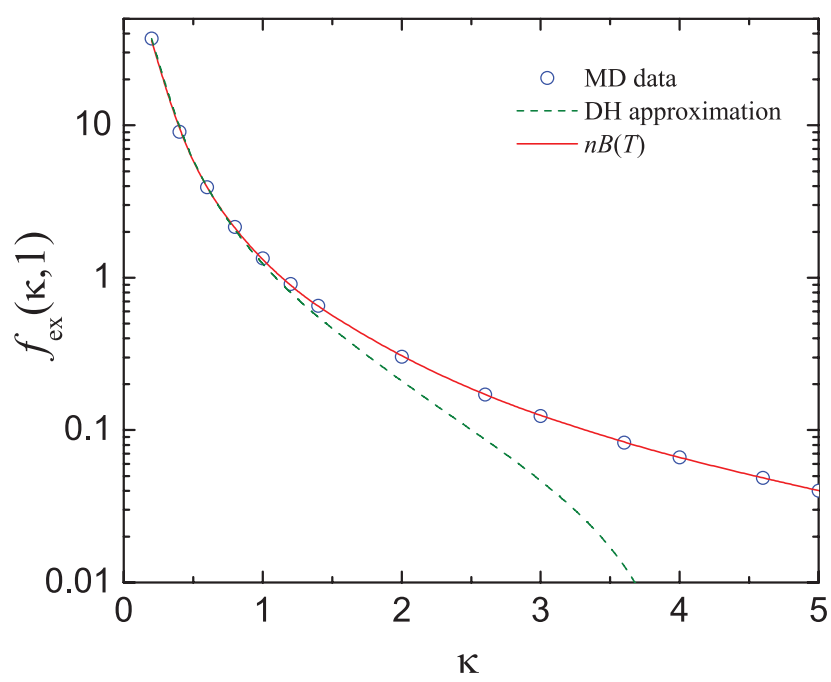

Figure 1. The excess free energy $f_{\mathrm{ex}}$ of the SCYS as a function of the screening parameter $\kappa$ for the fixed coupling parameter, $\Gamma=1$. Symbols are the results from MD simulations [17], the dashed line corresponds to the $\mathrm{DH}$ approximation, and the solid line has been calculated from the second virial coefficient, equation (7).

completely determined by particle-particle interactions and correlations. In real systems (e.g. complex plasmas or colloidal suspensions), the neutralizing medium is normally present to neutralize and stabilize like-charged particles. In this case, particle-medium interactions also affect thermodynamics. However, the effect of particle-medium interactions is additive to that of particle-particles interactions and can be easily evaluated [29]. For example, the excess (free) energy associated with the presence of neutralizing medium (e.g. plasma) is

$$
f_{\mathrm{pl}}=u_{\mathrm{pl}}=-\frac{3 \Gamma}{2 \kappa^{2}}-\frac{\kappa \Gamma}{2} .
$$

Note that the contribution of the neutralizing medium is negative and dominant at strong coupling, implying that the excess energy and pressure of the corresponding system are also negative in this regime. For the single component Yukawa system these quantities are obviously positive.

\section{Weakly coupled regime}

In the weakly coupled regime, the Debye-Hückel (DH) approach can be expected to provide reasonable accuracy in estimating thermodynamic properties of Yukawa systems. This is however true only near the one-component-plasma (OCP) limit (small $\kappa$ regime). As $\kappa$ increases, the DH approach (see e.g. [24] for explicit formulas) becomes progressively less and less accurate, and at certain $\kappa$ it even fails to predict correctly the sign of thermodynamic quantities. An example is shown in figure 1, where we plot the reduced excess (over that of noninteracting particles) free energy as a function of $\kappa$ at the fixed coupling parameter $\Gamma=1$. As $\kappa$ increases, the actual coupling weakens (since $\Gamma$ is fixed), but the $\mathrm{DH}$ approximation becomes completely irrelevant. The reason for that is the linearization of the Boltzmann factor, involved in the DH approach, which produces unphysical negative values of the particle 


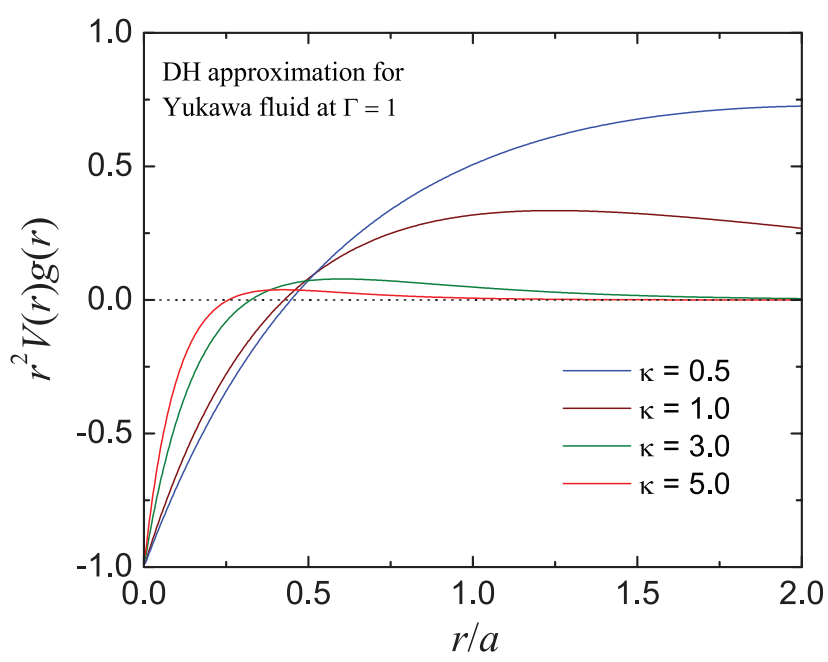

Figure 2. The factor $r^{2} V(r) g(r)$ appearing under the integral of the energy equation as a function of the reduced distance from the test particle, $r / a$. As $\kappa$ increases, the relative effect of the unphysical negative density region becomes progressively more and more important, causing the failure of the DH approximation.

density (or, equivalently, radial distribution function $g(r)$ ) in the vicinity of the test particle. Although, the spatial extent of this vicinity diminishes as $\kappa$ increases, its contribution to the thermodynamic quantities, such as the excess energy becomes progressively more and more significant. This is illustrated in figure 2, where the product $r^{2} V(r) g(r)$ appearing under the integral of the energy equation is plotted for several values of $\kappa$ at $\Gamma=1$. Thus, to some extent counter-intuitively, the $\mathrm{DH}$ approximation cannot be applied even in the regime of weak coupling for $\kappa \gtrsim 1$.

There is a known modification-the 'Debye-Hückel plus hole' (DHH) approach - to improve the performance of the $\mathrm{DH}$ approximation for the OCP [30]. The main idea is to truncate the exponential density in order to avoid negative values at small distances from the test particle after linearization. Recently, the original DHH scheme for the OCP has been generalized for Yukawa systems [24]. It can be particularly useful for weak and moderate coupling regimes. However, a much more simple and physically transparent approach to the thermodynamics of weakly coupled Yukawa systems is through the second virial coefficient. In this approximation, the excess free energy is expressed as [28]

$$
f_{\mathrm{ex}} \simeq 2 \pi n \int\left[1-\mathrm{e}^{-V(r) / T}\right] r^{2} \mathrm{~d} r .
$$

The values of $f_{\mathrm{ex}}(\kappa, 1)$ calculated from equation (7) are compared with the results from MD simulations [17] in figure 1. The agreement is rather good: at small $\kappa$ the deviations are within a few percents, at $\kappa \simeq 5$ they reduce to only two parts in one thousand.

\section{Strongly coupled fluids}

For strongly coupled systems, the reduced excess energy can be conveniently divided into the static and thermal components

$$
u_{\mathrm{ex}}=u_{\mathrm{st}}+u_{\mathrm{th}} \text {. }
$$

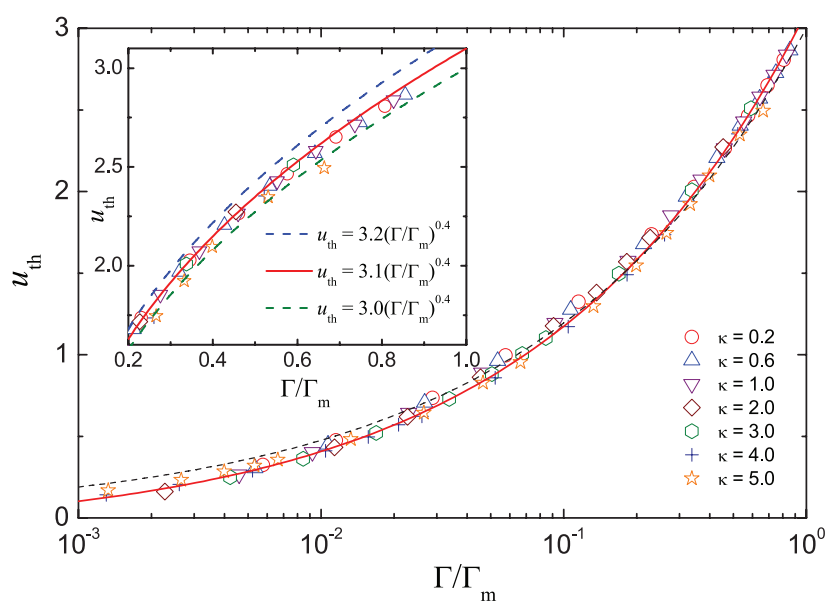

Figure 3. Thermal component of the reduced excess energy of Yukawa fluids versus the reduced coupling parameter $\Gamma / \Gamma_{\mathrm{m}}$. Symbols correspond to the numerical simulations for different values of the screening parameter $\kappa[17,35]$. The (red) solid curve is the fit of equation (10) with $\delta=3.2$ and $\epsilon=-0.1$. The (black) dashed line corresponds to the same functional form, but with $\delta=3.0$ and $\epsilon=0$, as suggested in [32]. The inset shows the same data in the near-freezing regime along with the convenient fits.

The static contribution corresponds to the value of internal energy when the particles are frozen in some regular configuration and the thermal corrections arise due to the deviations of the particles from these fixed position (due to thermal motion). Of course, such a division is only meaningful when the regular structure is specified. For crystals, the corresponding lattice sum is a relevant choice for $u_{\mathrm{st}}$. For fluids, it is convenient to link $u_{\text {st }}$ with the energy obtained using the Percus-Yevick (PY) radial distribution function of hard spheres in the unphysical limit $\eta=1$, where $\eta$ is the hard sphere packing fraction $[31,32]$. For the Yukawa system this is equivalent to the result of the ion sphere model (ISM), where each particle is placed in the center of the charge neutral Wigner-Seitz spherical cell and the energy is then calculated from simple electrostatic consideration [25]. With this choice, the static component of the internal energy of the single component Yukawa fluids becomes $[25,31,32]$

$$
u_{\mathrm{st}}=M_{\mathrm{f}}(\kappa) \Gamma=\frac{\kappa(\kappa+1) \Gamma}{(\kappa+1)+(\kappa-1) \mathrm{e}^{2 \kappa}},
$$

where $M_{\mathrm{f}}$ has been termed the fluid Madelung constant [32].

As Rosenfeld and Tarazona first pointed out, the thermal component of the internal energy exhibits quasi-universal behavior for a wide class of soft repulsive potentials (in particular, they considered the inverse-power-law and Yukawa potentials) in three dimensions [31, 32]. The accuracy of this scaling for various model systems has recently been investigated in extensive numerical simulations [33]. It has been observed that the RT scaling is particulary useful for liquids with strong correlations between equilibrium fluctuations of virial and potential energy [33], which are referred to as Rosklide-simple or just Rosklide systems [34]. The quality of the RT scaling for strongly coupled Yukawa systems is illustrated in figure 3 , where the dependence of $u_{\mathrm{th}}$ on $\Gamma / \Gamma_{\mathrm{m}}$ is plotted for a number of screening parameters, $\kappa<5$. To produce this 
plot, the numerical data on $u_{\mathrm{ex}}(\kappa, \Gamma)$ and $\Gamma_{\mathrm{m}}(\kappa)$ tabulated in $[17,35]$ have been used (the contribution of the neutralizing medium has been subtracted). It is seen that numerical data have a tendency to group around a single quasi-universal curve. Reasonably accurate fits (shown by the curves) can be obtained by using the following functional form

$$
u_{\mathrm{th}}=\delta\left(\Gamma / \Gamma_{\mathrm{m}}\right)^{2 / 5}+\epsilon .
$$

The original suggestion of Rosenfeld to use $\delta=3.0$ and $\epsilon=0$ [32] is shown by the dashed curve. Some improvement can be observed when choosing $\delta=3.2$ and $\epsilon=-0.1$, as documented in figure 3 . These values, suggested in [36], are therefore adopted here. In the near-freezing regime $\left(\Gamma / \Gamma_{\mathrm{m}} \gtrsim 0.2\right)$ the parameters $\delta=3.1$ and $\epsilon=0$ provide very good agreement with the numerical results, as shown in the inset [37]. Note that although the functional form (10) with the exponent $\frac{2}{5}$ provides reasonable accuracy for Yukawa fluids in a wide range of $\kappa$, it can be not the best choice for each single value of $\kappa$. For instance, in the case of OCP (limiting case $\kappa=0$ of Yukawa systems), the exponent $\frac{1}{3}$ is known to deliver better accuracy [35, 38-40]. Moreover, for Yukawa systems near the OCP limit $(\kappa \lesssim 1)$ the OCP scaling of the thermal energy is also superior to that of the RT scaling. This observation can be used to further improve the accuracy of estimating thermodynamic properties of weakly screened SCYS, as has been discussed in [41].

Equations (8)-(10) with the proper expression for $\Gamma_{\mathrm{m}}(\kappa)$ (see below) provide a simple and accurate tool to estimate the excess energy of Yukawa fluids. The excess free energy can then be obtained by the standard integration

$$
f_{\mathrm{ex}}(\kappa, \Gamma)=\int_{0}^{\Gamma} \frac{u_{\mathrm{ex}}\left(\kappa, \Gamma^{\prime}\right)}{\Gamma^{\prime}} \mathrm{d} \Gamma^{\prime} .
$$

Note that in case of the non-zero parameter $\epsilon$, this integral is diverging logarithmically. A simple conventional procedure to avoid this divergence is to start integration from $\Gamma=1$ in (11) and add the corresponding value $f_{\mathrm{ex}}(\kappa, 1)$ [17]. The values of $f_{\text {ex }}(\kappa, 1)$ have been tabulated in $[17,35]$, they can be also easily estimated from equation (7) above. However, the contribution from the weakly coupled region is in fact of very minor importance for $\Gamma \gg 1$ and can be omitted completely when the compressibility and related quantities at strong coupling are concerned [36].

The remaining step towards accurate estimation of thermodynamic properties of strongly coupled Yukawa fluids is to specify the functional dependence $\Gamma_{\mathrm{m}}(\kappa)$. Several approximate methods to locate the fluid-solid phase transition, based on the properties of the interaction potential alone, have been discussed in the literature [42-46]. Being focused on Yukawa interaction here, a simple expression proposed by Vaulina et al $[47,48]$ can be chosen. It reads

$$
\Gamma_{\mathrm{m}}(\kappa) \simeq \frac{172 \exp (\alpha \kappa)}{1+\alpha \kappa+\frac{1}{2} \alpha^{2} \kappa^{2}},
$$

where the constant $\alpha=(4 \pi / 3)^{1 / 3} \simeq 1.612$ is the ratio of the mean interparticle distance $\Delta=n^{-1 / 3}$ to the Wigner-Seitz radius $a$. Equation (12) is in rather good agreement with the numerical data in the regime $\kappa \lesssim 5$ addressed in this study. In the next section the explicit expressions for the compressibility factor and isothermal compressibility modulus are provided, which are convenient for practical calculations.

\section{Practical expressions for pressure and isother- mal compressibility modulus of Yukawa fluids}

Using the results of the previous section, the explicit expression for the compressibility factor (reduced pressure) can be derived [36]

$$
Z(\kappa, \Gamma)=\left(1+\frac{\epsilon}{3}\right)+\frac{\Gamma \kappa^{4}}{6[\kappa \cosh (\kappa)-\sinh (\kappa)]^{2}}+\frac{\delta}{3}\left(\frac{\Gamma}{\Gamma_{\mathrm{m}}}\right)^{2 / 5} f_{\mathrm{Z}}(\alpha \kappa),
$$

where

$$
f_{\mathrm{Z}}(x)=\frac{x^{3}+x^{2}+2 x+2}{x^{2}+2 x+2} .
$$

The isothermal compressibility modulus is related to the compressibility factor via [36] $\mu=Z+(\Gamma / 3)(\partial Z / \partial \Gamma)-(\kappa / 3)(\partial Z / \partial \kappa)$. This yields

$$
\mu(\kappa, \Gamma)=\left(1+\frac{\epsilon}{3}\right)+\frac{\Gamma \kappa^{6} \sinh (\kappa)}{9[\kappa \cosh (\kappa)-\sinh (\kappa)]^{3}}+\frac{\delta}{45}\left(\frac{\Gamma}{\Gamma_{\mathrm{m}}}\right)^{2 / 5} f_{\mu}(\alpha \kappa),
$$

where

$$
f_{\mu}(x)=\frac{2 x^{6}+14 x^{5}+35 x^{4}+76 x^{3}+136 x^{2}+136 x+68}{\left(x^{2}+2 x+2\right)^{2}} .
$$

The dependence $\Gamma_{\mathrm{m}}(\kappa)$ is given by equation (12); the recommended values of the coefficients $\delta$ and $\epsilon$ are: $\delta=3.2$ and $\epsilon=-0.1$.

The detailed comparison [36] between the compressibility factors obtained using equation (13) and those from direct MC simulations has demonstrated excellent performance of the proposed analytic formula.

\section{Example: sound velocity in complex (dusty) plasmas}

The effect of strong coupling on wave phenomena in complex (dusty) plasmas remains a very important current research topic [49-53]. Here we adopt the simplest fluid description of multi-component plasmas, similar to that used in the original derivation of DAW dispersion relation in [54]. In this formulation electrons and ions provide equilibrium neutralizing medium and are described by

$$
\begin{gathered}
-e n_{\mathrm{i}} \nabla \phi=T_{\mathrm{i}} \nabla n_{\mathrm{i}}, \\
e n_{\mathrm{e}} \nabla \phi=T_{\mathrm{e}} \nabla n_{\mathrm{e}},
\end{gathered}
$$

where $\phi$ is the electric potential, $n_{\mathrm{e}, \mathrm{i}}$ and $T_{\mathrm{e}, \mathrm{i}}$ are the density and temperature of electrons and ions, respectively. Equations (17) and (18) result in equilibrium Boltzmann relations for the ion and electron densities in the wave potential. The continuity and momentum equations for the particles are 


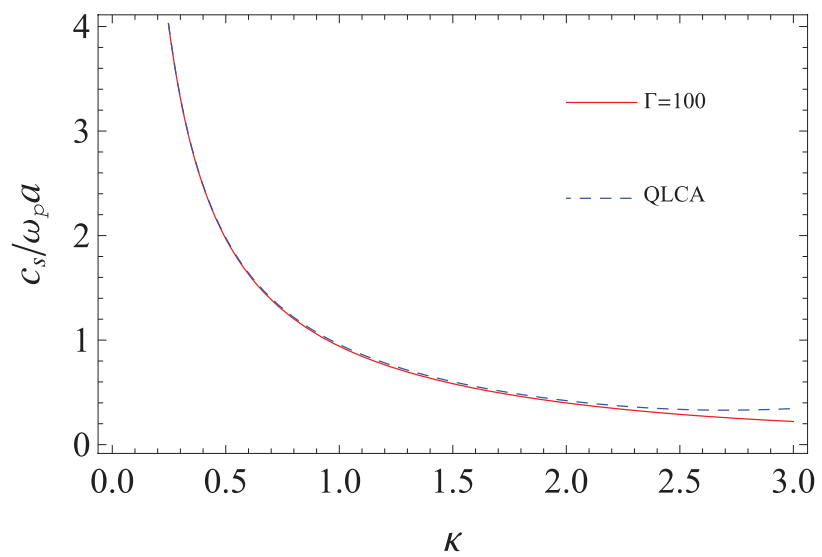

Figure 4. Reduced sound velocity of Yukawa fluids, $c_{\mathrm{s}} / \omega_{\mathrm{p}} a$, as a function of the screening parameter $\kappa$. The solid curve corresponds to the results of the simple fluid approach of this section for $\Gamma=100$. The dashed curve is plotted using QLCA result of [58].

$$
\begin{gathered}
\frac{\partial n}{\partial t}+\nabla(n \mathbf{v})=0, \\
\frac{\partial \mathbf{v}}{\partial t}+(\mathbf{v} \cdot \nabla) \mathbf{v}=-\frac{Q \nabla \phi}{m_{\mathrm{p}}}-\frac{\nabla P}{m_{\mathrm{p}} n},
\end{gathered}
$$

where $\mathbf{v}$ is the particle velocity, $m_{\mathrm{p}}$ is the particle mass, and $P$ is the pressure associated with the particle component. In the limit of long-wavelength perturbations (acoustic regime) the system is quasineutral,

$$
e n_{\mathrm{i}}-e n_{\mathrm{e}}+Q n=0 .
$$

The standard linearization procedure applied to the set of equations (17)-(21) results in the dispersion relation of the acoustic type

$$
c_{\mathrm{s}}^{2} \equiv \frac{\omega^{2}}{k^{2}}=\omega_{\mathrm{p}}^{2} \lambda_{\mathrm{D}}^{2}+v_{\mathrm{Tp}}^{2} \gamma \mu,
$$

where $c_{\mathrm{s}}$ is the sound velocity, $\omega_{\mathrm{p}}=\sqrt{4 \pi Q^{2} n / m_{\mathrm{p}}}$ is the plasma-particle frequency, $\lambda_{\mathrm{D}}$ is the linearized Debye radius, $v_{\mathrm{Tp}}=\sqrt{T / m_{\mathrm{p}}}$ is the particle thermal velocity, $\gamma=C_{\mathrm{P}} / C_{\mathrm{V}}$ is the adiabatic index, $\mu=(1 / T)(\partial P / \partial n)_{T}$ is the isothermal compressibility modulus, $\omega$ is the wave frequency, and $k$ is the wave number. Rewritten in terms of the reduced Yukawa state variables, $\kappa$ and $\Gamma$, the dispersion relation (22) becomes

$$
c_{\mathrm{s}}=\omega_{\mathrm{p}} a\left(\frac{1}{\kappa^{2}}+\frac{\gamma \mu}{3 \Gamma}\right)^{1 / 2} .
$$

The remaining step to identify the influence of strong coupling on the sound velocity is to take the appropriate values for $\gamma$ and $\mu$. Note at this point, that by way of derivation the quantities $\gamma$ and $\mu$, appearing in equation (23) account for both, particle-particle correlations and neutralizing plasma effects. However, as shown in [55], the only plasma-related contribution to the product $\gamma \mu$ that matters cancels out exactly the plasma contribution to the dispersion relation (term $1 / \kappa^{2}$ in equation (23)). Thus, the sound velocity of a system of charged particles in the neutralizing medium is equal to that of the

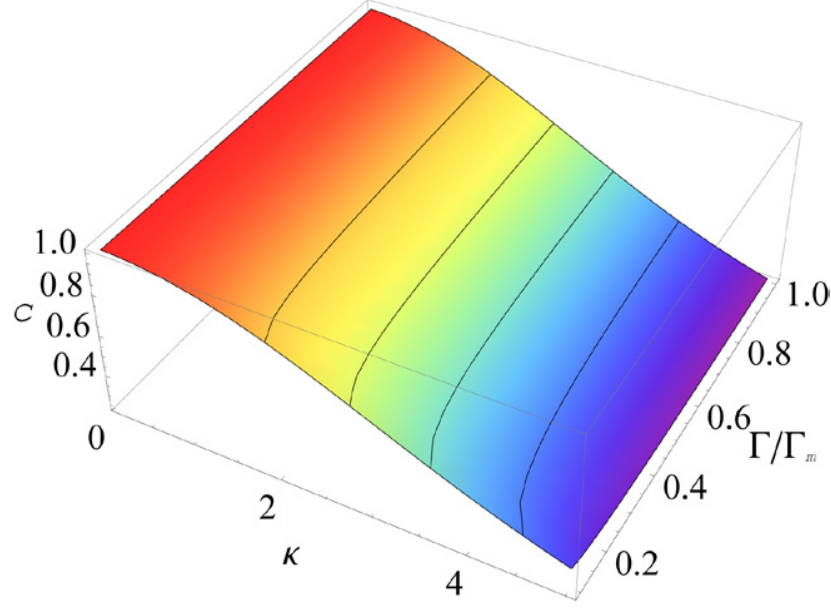

Figure 5. Three-dimensional plot of the reduced sound velocity $c \equiv c_{\mathrm{s}} / c_{0}$ as function of Yukawa system state variables $\kappa$ and $\Gamma / \Gamma_{\mathrm{m}}$. Calculations are made using the fluid model described in this paper.

SCYS. The standard expression for single-component fluids [56], $c_{\mathrm{s}}=v_{\mathrm{Tp}} \sqrt{\gamma \mu}$, is applicable. In terms of the quantities derived above, the sound velocity is

$$
c_{\mathrm{s}}=\omega_{\mathrm{p}} a\left(\frac{\mu}{3 \Gamma}+\frac{[Z-\Gamma(\partial Z / \partial \Gamma)]^{2}}{3 \Gamma[u-\Gamma(\partial u / \partial \Gamma)]}\right)^{1 / 2},
$$

where now $\mu, Z$, and $u$ all correspond to SCYS, that is analytic expressions from the previous sections can be used for calculation.

First, the relevance of this simple fluid approach can be checked using the existing results from the quasi-localized charge approximation (QLCA) model [57-59]. An example of such a comparison is shown in figure 4 , where the reduced sound velocity is plotted as a function of the screening parameter $\kappa$. The curve corresponding to the present approach is practically indistinguishable from the QLCA result of [58]. Good agreement of the present approach with the long-wavelength part of the longitudinal dispersion relation of Yukawa fluids obtained in a numerical (MD) experiment [60] has also been documented in [55].

Having demonstrated good agreement between the present fluid analysis and previous results from the QLCA model and MD simulations, let us investigate the dependence of the sound velocity on coupling and screening in detail. It is particularly useful to analyze the behavior of the quantity $c_{\mathrm{s}} / c_{0}$, which is the ratio of the actual sound velocity of a Yukawa fluid to the respective limiting 'ideal gas' (weak coupling limit) value given by

$$
c_{0}=\omega_{\mathrm{p}} \lambda_{\mathrm{D}}
$$

The $3 \mathrm{D}$ plot of this quantity in the $\left(\kappa, \Gamma / \Gamma_{\mathrm{m}}\right)$ plane is shown in figure 5. First, we observe only very weak dependence of the quantity $c_{\mathrm{s}} / c_{0}$ on $\Gamma$ deep in the fluid regime (on approaching the fluid-solid phase transition). This implies that the absolute value of $c_{\mathrm{s}}$ increases with $\Gamma$, because $c_{0} \propto \sqrt{\Gamma}$. Second, we observe that the ratio $c_{\mathrm{s}} / c_{0}$ is sensitive to the screening parameter, and decreases as $\kappa$ increases. It drops by almost one order 
of magnitude on the way from the weakly screened regime $\kappa \lesssim 1$ to the strongly screened regime with $\kappa \simeq 5$.

\section{Yukawa solids}

The reduced excess energy of a solid in the harmonic approximation is

$$
u_{\mathrm{s}}=M_{\mathrm{s}} \Gamma+\frac{3}{2}
$$

where $M_{\mathrm{s}}$ is the corresponding lattice sum (Madelung constant). The reduced Helmholtz free energy of a solid is related to the excess energy by the standard integration (equation (4)), which yields

$$
f_{\mathrm{s}}=M_{\mathrm{s}}\left(\Gamma-\Gamma_{\mathrm{m}}\right)+\frac{3}{2} \ln \left(\Gamma / \Gamma_{\mathrm{m}}\right)+f_{\mathrm{m}} .
$$

Here the integration starts from $\Gamma_{\mathrm{m}}$ and $f_{\mathrm{m}}=f\left(\Gamma_{\mathrm{m}}\right)$. According to the Ross melting criterion [61], which he obtained by reformulating the celebrated Lindemann melting law in terms of the statistical-mechanical partition function

$$
f_{\mathrm{m}} \simeq M_{\mathrm{s}} \Gamma_{\mathrm{m}}+\mathcal{C}
$$

where $\mathcal{C}$ is some constant. In other words, the thermal component (entropy) of the reduced excess free energy remains approximately constant at melting. In fact, $\mathcal{C}$ somewhat varies with the variation of the potential softness, but these variations remain relatively week, with $\mathcal{C} \simeq 6$ for a rather broad range of interaction potentials investigated $[62,63]$.

The values of $\mathcal{C}$ at melting of the bcc and fcc Yukawa solids have been calculated, in the harmonic lattice approximation, using the data tabulated in $[17,64]$. In addition to neglecting anharmonic corrections, one can use the fact that the fluid-bcc and fluid-fcc transition lines are very close to each other in the parameter regime of present interest (for the confirmation see figure 7 of [19]), so that a unique value of $\Gamma_{m}$ can be used (this is further justified by the fact that $\Gamma_{\mathrm{m}}$ appears under the logarithm when calculating $\mathcal{C}$ ). The obtained values of $\mathcal{C}$ can be approximated as [37]

$$
\mathcal{C}(\kappa)= \begin{cases}6.55-0.13 \kappa, & (\text { bcc lattice }) \\ 6.57-0.10 \kappa & (\text { fcc lattice })\end{cases}
$$

The resulting expression for the reduced free energy of the solid phase is

$$
f_{\mathrm{s}}(\kappa, \Gamma)=M_{\mathrm{s}}(\kappa) \Gamma+\frac{3}{2} \ln \left[\Gamma / \Gamma_{\mathrm{m}}(\kappa)\right]+\mathcal{C}(\kappa) .
$$

To use equation (29) for practical evaluations the dependence $\Gamma_{\mathrm{m}}(\kappa)$ given by equation (12) can be employed.

Equation (5) complemented by equations (28)-(29) has been used to evaluate the compressibility of Yukawa solids in a wide regime of parameters in [37]. The results have been compared with direct MC [16] and MD [37] simulations as well as with the recently proposed shortest-graph method $[65,66]$. Very good overall agreement has been documented. For most of the state points, the relative deviations from the accurate (MC or MD) results are well below one part in one thousand.
Given the simplicity of the proposed theoretical approach, such an agreement should be considered as excellent.

\section{Concluding remarks}

We have discussed simple, but rather accurate approaches to evaluate thermodynamic properties of Yukawa fluids and solids across coupling regimes. These approaches can be particularly useful in the context of complex plasmas and colloidal suspensions. One example, related to the evaluation of the sound velocity in complex plasmas (assuming purely Yukawa interaction between the particles) has been presented. Several important remarks should be added in conclusion.

This paper considered only three-dimensional systems, although two-dimensional systems are also of considerable interest. There exists extensive literature on the thermodynamics of two-dimensional Yukawa systems, which has not been included in the present reference list. Recently, it was evidenced that the thermal component of the internal energy exhibits quasi-universal behavior also in two-dimensional systems of soft repulsive particles [41]. In particular, the functional dependence of the thermal energy of the two-dimensional OCP can be adopted for weakly screened Yukawa systems [41]. Thus, the approaches discussed above can be also applied, with some modifications, to the two-dimensional Yukawa systems.

Thermodynamic properties of Yukawa fluids (weakly and strongly coupled), and solids have been addressed. Recently, there was also considerable interest in understanding glass-transition properties for Yukawa (and modified-Yukawa) potentials. The location of glass-transition lines on the phase diagrams of related systems has been identified for both, two- and threedimensional systems. The details can be found in [67-69].

The approaches discussed in this paper are based on simple phenomenological arguments, which are likely applicable to a wide class of soft repulsive interactions. One relevant example deals with inverse-power-law interactions, $V(r) \propto r^{-\alpha}$, for which the RT scaling holds (for $\alpha \lesssim 5$ [37]). It can be expected, therefore, that the discussed approaches can also be helpful in connection with the thermodynamics of soft matter systems exhibiting water-like anomalies (Gaussian core, exp-6, Hertz and related model potentials) in the regime of sufficiently soft interaction (high density), but this requires proper verification.

The last remark deals with the applicability of the simple Yukawa model discussed here to real systems, like for instance complex plasmas. In particular, significant limitations can be related to the openness of the complex plasma systems. Plasma electrons and ions are continuously lost on the particle surface and the particle charge is set by the condition of no net electrical current to the surface (or, equivalently, floating potential at the particle surface). This is known to result in some deviations from the Yukawa-type potential around the particles, as has been discussed in the Introduction. These, in turn, can lead to some deviations from the thermodynamic functions of conventional Yukawa systems. Perhaps even more important is that the particle charge in complex plasmas is not fixed, but depends on the parameters of the surrounding 
plasma. In particular, the charge becomes a function of the particle density via the so called 'charge cannibalism' effect [70-72]. This effect operates as follows: when the particle density increases, the negative charge carried by the particle component also increases, which results in some reduction of the electron-to-ion density ratio (electron depletion) in view of the quasineutrality condition. In turn, this suppresses the efficiency of electron collection by the particle surface compared to that of the ions. The particle charge becomes less negative, i.e. decreases in the absolute magnitude compared to the case of an individual particle. In general, the relation between the particle charge and number density and the densities of electrons and ions in complex plasmas is governed by the quasineutrality condition and the competition between specific plasma production and loss mechanisms operating in a given situation. All this indicates that the consideration of an idealized Yukawa system with fixed particle charges and background plasma density can be in many cases insufficient to mimic the actual thermodynamics of real complex plasmas. How large modifications can be and whether they can be evaluated using conventional thermodynamic approaches require careful investigation.

\section{Acknowledgments}

This work has been supported by the A*MIDEX grant (Nr. ANR-11-IDEX-0001-02) funded by the French Government 'Investissements d'Avenir' program. I would like to thank my colleagues, L Couedel, A Ivlev, A Khrapak, N Kryuchkov, G Morfill, I Semenov, H Thomas, and S Yurchenko who participated at different stages of this project. Cooperation with A G Khrapak was supported by the Russian Foundation for Basic Research, Project No. 13-02-01099. I thank Dominique Escande for reading this manuscript and suggesting improvements.

\section{References}

[1] Ivlev A, Löwen H, Morfill G and Royall C P 2012 Complex Plasmas and Colloidal Dispersions: Particle-Resolved Studies of Classical Liquids and Solids (Singapore: World Scientific)

[2] Fortov V E, Khrapak A G, Khrapak S A, Molotkov V I and Petrov O F 2004 Phys.-Usp. 47447

Fortov V E, Ivlev A V, Khrapak S A, Khrapak A G and Morfill G E 2005 Phys. Rep. 4211

[3] Chaudhuri M, Ivlev A V, Khrapak S A, Thomas H M and Morfill G E 2011 Soft Matter 71287

[4] Allen J E, Annaratone B M and de Angelis U 2000 J. Plasma Phys. 63299

[5] Zobnin A V, Nefedov A P, Sinel'shchikov V A and Fortov V E 2000 J. Exp. Theor. Phys. 91483

[6] Khrapak S A, Klumov B A and Morfill G E 2008 Phys. Rev. Lett. 100225003

[7] Khrapak S and Morfill G 2009 Contrib. Plasma Phys. 49148

[8] Semenov I L, Zagorodny A G and Krivtsun I V 2011 Phys. Plasmas 18103707

[9] Daugherty J E, Porteous R K, Kilgore M D and Graves D B 1992 J. Appl. Phys. 723934

[10] Hutchinson I H and Haakonsen C B 2013 Phys. Plasmas 20083701
[11] Semenov I L, Khrapak S A and Thomas H M 2015 Phys. Plasmas 22053704

[12] Lampe M, Goswami R, Sternovsky Z, Robertson S, Gavrishchaka V, Ganguli G and Joyce G 2003 Phys. Plasmas 101500

[13] Khrapak S A, Ivlev A V and Morfill G E 2010 Phys. Plasmas 17042107

[14] Wysocki A et al 2010 Phys. Rev. Lett. 105045001

[15] Robbins M O, Kremer K and Grest G S 1988 J. Chem. Phys. 883286

[16] Meijer E J and Frenkel D 1991 J. Chem. Phys. 942269

[17] Hamaguchi S, Farouki R T and Dubin D H E 1997 Phys. Rev. E 564671

[18] Caillol J M and Gilles D 2000 J. Stat. Phys. 100933

[19] Tejero C F, Lutsko J F, Colot J L and Baus M 1992 Phys. Rev. A 463373

[20] Faussurier G 2004 Phys. Rev. E 69066402

[21] Tolias P, Ratynskaia S and de Angelis U 2014 Phys. Rev. E 90053101

[22] Totsuji H 2008 Phys. Plasmas 15072111

[23] Vaulina O S, Koss X G, Khrustalev Y V, Petrov O F and Fortov V E 2010 Phys. Rev. E 82056411

[24] Khrapak S A, Khrapak A G, Ivlev A V and Morfill G E 2014 Phys. Rev. E 89023102

[25] Khrapak S A, Khrapak A G, Ivlev A V and Thomas H M 2014 Phys. Plasmas 21123705

[26] Khrapak S A and Khrapak A G 2015 Phys. Plasmas 22044504

[27] Khrapak A G and Khrapak S A 2015 AIP Adv. 5087175

[28] Landau L D and Lifshitz E M 2005 Statistical Physics (Amsterdam: Elsevier)

[29] Hamaguchi S and Farouki R T 1994 J. Chem. Phys. 1019876

[30] Nordholm S 1984 Chem. Phys. Lett. 105302

[31] Rosenfeld Y and Tarazona P 1998 Mol. Phys. 95141

[32] Rosenfeld Y 2000 Phys. Rev. E 627524

[33] Ingebrigtsen T S, Veldhorst A A, Schrøder T B and Dyre J C 2013 J. Chem. Phys. 139171101

[34] Batcher A K, Schrøder T B and Dyre J C 2014 Nat. Commun. 55424

[35] Farouki R T and Hamaguchi S 1994 J. Chem. Phys. 1019885

[36] Khrapak S A and Thomas H 2015 Phys. Rev. E 91023108

[37] Khrapak S A, Kryuchkov N P, Yurchenko S O and Thomas H M 2015 J. Chem. Phys. 142194903

[38] Stringfellow G S, DeWitt H E and Slattery W L 1990 Phys. Rev. A 411105

[39] Dubin D H E and O’Neil T M 1999 Rev. Mod. Phys. 7187

[40] Khrapak S A and Khrapak A G 2014 Phys. Plasmas 21104505

[41] Khrapak S A, Semenov I, Couedel L and Thomas H 2015 Phys. Plasmas 22083706

[42] Rosenfeld Y 1976 J. Chem. Phys. 641248

Rosenfeld Y 1976 Mol. Phys. 32963

[43] Khrapak S A and Morfill G E 2009 Phys. Rev. Lett. 103255003

[44] Khrapak S A, Chaudhuri M and Morfill G E 2010 Phys. Rev. B 82052101

Khrapak S A, Chaudhuri M and Morfill G E $2011 \mathrm{~J}$. Chem. Phys. 134054120

[45] Khrapak S A, Chaudhuri M and Morfill G E 2011 J. Chem. Phys. 134241101

[46] Khrapak S A and Saija F 2011 Mol. Phys. 1092417

[47] Vaulina O S and Khrapak S A 2000 J. Exp. Theor. Phys. 90287

[48] Vaulina O, Khrapak S and Morfill G 2002 Phys. Rev. E 66016404

[49] Merlino R L 2014 J. Plasma Phys. 80773

[50] Salin G 2007 Phys. Plasmas 14082316

[51] Yaroshenko V V, Nosenko V and Morfill G E 2010 Phys. Plasmas 17103709 
[52] Avinash K, Merlino R L and Shukla P K 2011 Phys. Lett. A 3752854

[53] Rosenberg M, Kalman G J, Hartmann P and Goree J 2014 Phys. Rev. E 89013103

[54] Rao N N, Shukla P K and Yu M Y 1990 Planet. Space Sci. 38543

[55] Khrapak S A and Thomas H M 2015 Phys. Rev. E 91033110

[56] Landau L D and Lifshitz E M 1987 Fluid Mechanics (Oxford: Pergamon)

[57] Golden K I and Kalman G J 2000 Phys. Plasmas 714

[58] Kalman G, Rosenberg M and DeWitt H E 2000 Phys. Rev. Lett. 846030

[59] Donko Z, Kalman G J and Hartmann P 2008 J. Phys.: Condens. Matter 20413101

[60] Ohta H and Hamaguchi S 2000 Phys. Rev. Lett. 846026

Hamaguchi S and Ohta H 2001 Phys. Scr. T89 127

[61] Ross M 1969 Phys. Rev. 184233

[62] Hoover W G, Gray S G and Johnson K W 1971 J. Chem. Phys. 551128
[63] Agrawal R and Kofke D A 1995 Mol. Phys. 8523

[64] Hamaguchi S, Farouki R T and Dubin D H E 1996 J. Chem. Phys. 1057641

[65] Yurchenko S O 2014 J. Chem. Phys. 140134502

[66] Yurchenko S O, Kryuchkov N P and Ivlev A V 2015 J. Chem. Phys. 143034506

[67] Sciortino F, Mossa S, Zaccarelli E and Tartaglia P 2004 Phys. Rev. Lett. 93055701

[68] Yazdi A, Ivlev A, Khrapak S, Thomas H, Morfill G E, Löwen H, Wysocki A and Sperl M 2014 Phys. Rev. E 89063105

[69] Yazdi A, Heinen M, Ivlev A, Löwen H and Sperl M 2015 Phys. Rev. E 91052301

[70] Havnes O, Morfill G E and Goertz C K 1984 J. Geophys. Res. 89999

[71] Barkan A, D’Angelo N and Merlino R L 1994 Phys. Rev. Lett. 733093

[72] Khrapak S A, Thomas H M and Morfill G E 2010 Europhys. Lett. 9125001 